\title{
Time-domain photocurrent spectroscopy based on a common-path birefringent interferometer
}

\author{
Lukas Wolz ${ }^{+},{ }^{1}$ Constantin Heshmatpour ${ }^{+}, 2$ Antonio Perri, ${ }^{3,4}$ Dario Polli, ${ }^{3,4}$ Giulio Cerullo, ${ }^{3,4}$ Jonathan J. \\ Finley, ${ }^{1}$ Erling Thyrhaug, ${ }^{2}$ Jürgen Hauer, ${ }^{2}$, a) and Andreas V. Stier ${ }^{1, b)}$ \\ 1) Walter Schottky Institut, Department of Physics, Technical University of Munich, 85748 Garching, \\ Germany \\ ${ }^{2)}$ Dynamical Spectroscopy, Department of Chemistry, Technical University of Munich, 85748 Garching, \\ Germany \\ 3) IFN-CNR and Dipartimento di Fisica, Politecnico di Milano, Piazza L. da Vinci 32, 20133 Milano, \\ Italy \\ ${ }^{4)}$ NIREOS S.R.L., Via G. Durando 39, 20158 Milano, Italy \\ ${ }^{+}$These authors contributed equally
}

(Dated: 10 October 2020)

We present diffraction-limited photocurrent (PC) microscopy in the visible spectral range based on broadband excitation and an inherently phase-stable common-path interferometer. The excellent path-length stability guarantees a high accuracy without the need for active feedback or post-processing of the interferograms. We illustrate the capabilities of the setup by recording PC spectra of a bulk GaAs device, and compare the results to optical transmission data.

\section{INTRODUCTION}

Photocurrent (PC) spectroscopy is a key absorption method for the investigation of light-matter interactions and carrier transport in optically active materials ${ }^{1,2}$. The broad range of applications includes characterization of semiconductor band structures ${ }^{1,3,4}$, excitons ${ }^{5}$, defect bands ${ }^{6}$ and the measurement of charge collection efficiency of artificial $^{7,8}$ and biological $^{9}$ nanoscale devices. Conventionally, PC spectra are recorded in the frequency domain with a tunable (quasi-) monochromatic source. An alternative interferometric implementation is time-domain Fourier Transform (FT) spectroscopy ${ }^{10}$. Time-domain spectroscopy features several advantages over frequency-domain approaches. The most notable in the present context are the well-known Jacquinot ${ }^{11}$ and Connes ${ }^{12}$ advantages. These state, respectively, that relative to a monochromator-based implementation, an interferometry based spectrometer will have a larger signal-to-noise ratio due to increased optical throughput and will feature better wavelength-accuracy due to a smaller reliance on accurate optical alignment. In the context of spectroscopy of semiconductor devices and photovoltaics, we also note that interferometer-based spectrometers tend to be substantially faster, since the collection times are independent of the measured wavelength interval - instead being purely a function of the desired spectral resolution. While FT photocurrent spectroscopy is advantageous in the infrared spectral range, where light sources are weak and optical features are typically broad, it has been much more difficult to implement in the visible range due to the significantly higher demands for interferometric phase-stability. In particular, it is difficult to achieve the desired phase stability of $\lambda / 100$ in conventional amplitude division interferometers such as Michelson or Mach-Zehnder geometries. Applications in the visible,

\footnotetext{
a) Juergen.Hauer@tum.de

b)Andreas.Stier@wsi.tum.de
}

based on these designs, thus typically require active phasestabilization or monitoring of pathlength-fluctuations by copropagation with a tracer beam. As an alternative to conventional (e.g. Mach-Zehnder) interferometers we present a photocurrent microscope based on the inherently phase-stable Translating-Wedge-based Identical pulses eNcoding System (TWINS) $)^{13}$ common-path interferometer. Instead of introducing path-length differences for separate replicas, TWINS achieves the desired phase delay $\tau$ by the propagation of a single polarized beam through an adjustable amount of birefringent material, resulting in two collinear, orthogonally polarized beams with a precisely controllable relative time-delay. The TWINS has been implemented successfully for bulk samples in several linear and non-linear interferometry applications in the infra-red ${ }^{14}$, visible ${ }^{15,16}$ and $\mathrm{UV}^{17}$ spectral ranges. Furthermore, we recently used it in demonstration of simultaneous collection of excitation and emission spectra of single molecules ${ }^{18}$. Here, we further extend the versatility of TWINS interferometry by recording spatially resolved timedomain photocurrent spectra in the visible spectral range. As a test sample, we use a simple biased-GaAs photodetector at room temperature. To validate the accuracy of the suggested setup, we compare the photocurrent spectrum retrieved by TWINS to spectra recorded after placing bandpass filters with known properties into the beam path.

\section{EXPERIMENTAL SETUP}

The TWINS interferometer (NIREOS, model Gemini) has been described in detail elsewhere ${ }^{13,19}$, and here we only briefly outline its operation principle in Figure 1 (a). An excitation beam is linearly polarized (Pol1) at $45^{\circ}$ relative to the optical axis of the birefringent Block A ( $\alpha$ barium borate $(\alpha-$ $\mathrm{BBO})$ ). This results in a pair of equally intense and orthogonally polarized components with a fixed phase delay between them due to the birefringence of the crystal. This phase delay can be compensated with a pair of $\alpha$-BBO wedges mounted 
on a linear motorized stage (Block B) as to allow precise control of the effective thickness $d_{B}(x)$ that the excitation light traverses. Note that the crystal axis of Block B is aligned at $90^{\circ}$ to block A. Thus, after transmission of the two blocks,

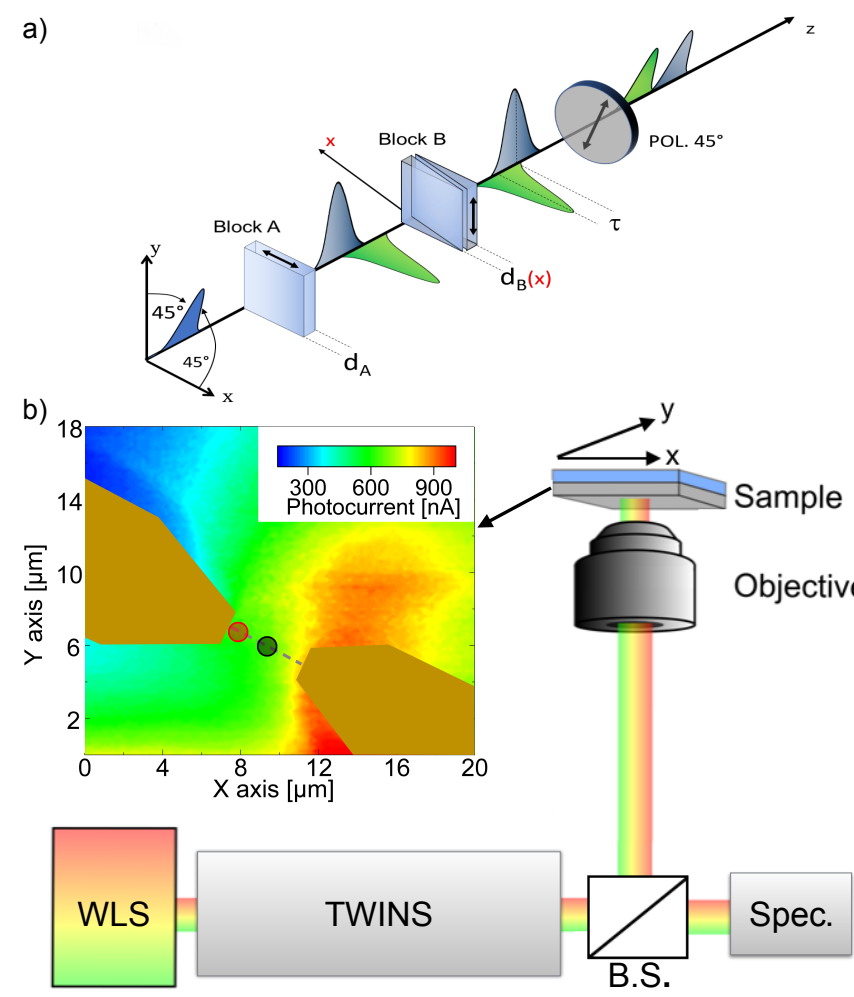

FIG. 1. (a) Illustration of the TWINS interferometer used in this experiment (NIREOS, model Gemini). The excitation light is linearly polarized at $\Theta=45^{\circ}$, with respect to the optical axis of the birefringent $\alpha$-barium borate crystal. Block A induces a fixed phase delay $\tau_{A}$ between the ordinary and extraordinary polarizations. Block B is made of a pair of $\alpha$-BBO wedges, which can be moved with respect to each other to induce a variable phase delay $-\tau_{B}(x)$. (b) Schematic of the TWINS photocurrent setup. A white-light source is collimated through the TWINS-interferometer and either fiber-coupled into a spectrometer or focused $(100 \mathrm{x}$ objective, $N A=0.7)$ on the photodetector. It consists of a pair of biased gold contacts, evaporated on top of bulk GaAs with a $4 \mu \mathrm{m}$ gap between them. The photocurrent is detected via 2-point measurement by a Keysight B2912A source meter. The contacts and the spot size are indicated in the PC map. The dashed line indicates a line scan discussed in the text.

the total phase delay $\tau(x)$ between ordinary and extraordinary polarization components is:

$$
\tau(x)=\tau_{A}-\tau_{B}(x)=\left(d_{A}-d_{B}(x)\right)\left(\frac{1}{v_{o r}}-\frac{1}{v_{e x}}\right)
$$

where $v_{o r}$ and $v_{e x}$ are the ordinary and extraordinary phase velocities, respectively. Ultimately, the resolution and precision of the interferometer rely solely on the precise movement and range of the wedges. With the typical $100 \mathrm{~nm}$ precision of the linear stage, we achieve $\sim 5$ attoseconds phase-delay accuracy at $500 \mathrm{~nm}$ and $0.8 \mathrm{ps}$ total phase delay for a scanning range of the wedges $\pm 12 \mathrm{~mm}$. After passing through the birefringent blocks, Pol2 projects both orthogonal components of the beam into the same polarization state, resulting in the interference of the two polarization components, depending on the total phase-delay. The configuration of the TWINS has the following advantages: $i$ ) being a common-path instrument, the phase-delay is - in contrast to e.g. standard Michelson interferometers - insensitive to mechanical fluctuations, and no feedback stabilization or position tracking is required ${ }^{20}$; and ii) due to the small apex angle of the wedges and the relatively small difference between the refractive indexes of ordinary and extraordinary polarization, the device demultiplies the transverse translation of the wedges, allowing extremely high delay accuracy. The TWINS-photocurrent microscope is schematically shown in Figure 1 (b). As excitation source, the collimated output of a pulsed supercontinuum laser (Fianium, WLS micro, $30 \mathrm{MHz}$ repetition rate) is directed through the TWINS interferometer and focused into a diffraction-limited spot on the photodetector. The total excitation power impinging on the photodetector is estimated by a silicon-based powermeter to be $4-5 \mu \mathrm{W}$ for all our measurements. The device is mounted on a piezoelectric XYZ stage, allowing (raster) scanning of the sample to yield a spatially resolved photocurrent (PC) map, as illustrated on the left side of Figure 1 (b). The device is connected to a break-out box, and the $\mathrm{PC}$ is recorded with a Keysight B2912A source/measure unit (SMU).

\section{RESULTS}

To demonstrate the scanning microscopy part of the TWINS-PC setup, we measured the photocurrent of a selfmade bulk GaAs photodetector, shown in the left side of Figure 1 (b) for a fixed wedge position $(\tau(x)=0)$. Thus the PC map is recorded under modulated "white-light" illumination (see Figure 2 (a), wedge position $=0$ ). The simple GaAs photodetector consists of two gold contacts defined on the surface of the semiconductor using standard e-beam evaporation and optical lithography techniques. The gap between the contacts was $4 \mu \mathrm{m}$ across which a bias voltage $\left(V_{\text {bias }}\right)$ was applied. The resulting photocurrent as a function of the excitation spot position is shown in the false-colour map in Figure 1 (b). Along the indicated line scan, the amplitude of the photocurrent follows the expected potential drop across the contacts (discussed in detail below).

We calibrated the TWINS-PC setup by fiber-coupling the excitation light into a spectrometer (Ocean Optics, HR4000) and recording the spectra for each wedge position. The recorded data for the range of $x=-0.4 \mathrm{~mm}$ to $x=0.4 \mathrm{~mm}$ are shown in the false-colour plot Figure 2 (a). Clearly, the observed intensity is modulated depending on wedge position and wavelength. The sinusoidal intensity modulation as a function of wedge position is shown for two wavelengths in Figure 2 (b). As we expect an intensity modulation with a $\lambda / 2$-periodicity, the spatial oscillatory period is approximately twice as long for $850 \mathrm{~nm}$ as compared to the line cut at $450 \mathrm{~nm}$. Fourier transforming these modulated signals at each wavelength (representative examples shown in Figure 2 
(c)) results in a single peak at a pseudo frequency (inverse wedge position, $\mathrm{mm}^{-1}$ ). A direct correlation between the inverse wedge position and wavelength, results in the fully constructed calibration curve shown in Figure 2 (d).
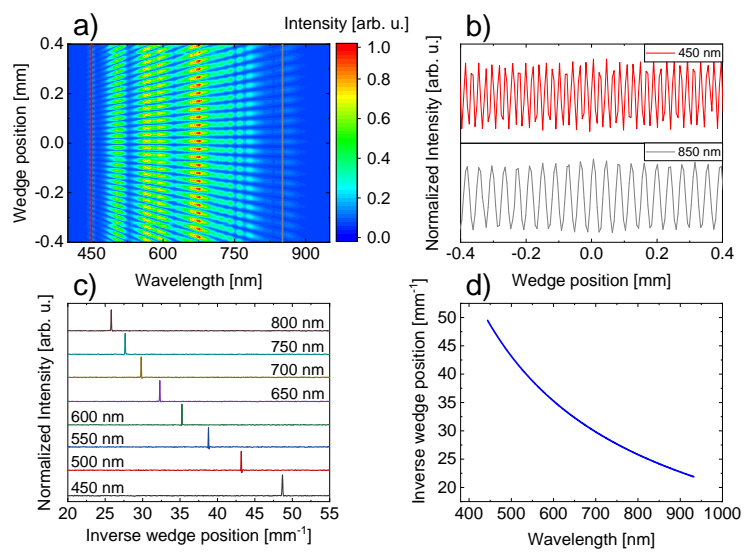

FIG. 2. (a) Colour map of the modulated spectral intensity close to zero phase delay $(\tau(\mathrm{x})=0)$. (b) Vertical line cuts through the spectral intensity shown in (a) at $\lambda=850 \mathrm{~nm}$ and $\lambda=450 \mathrm{~nm}$ showing the sinusoidal intensity modulation as a function of wedge position. (c) Waterfall plot of the Fourier-transforms of the modulated spectral intensity at a variety of fixed wavelengths. The Fourier-transform relates the modulation frequency of a specific wavelength to a spatial coordinate linked to the wedge position. (d) Correlation between the measured wavelength and the spatial coordinate obtained from the Fourier transform of the data shown in (a).

We test the calibration procedure of the setup by measuring the photocurrent spectra of our GaAs detector. With a bias voltage of $V_{\text {bias }}=8 \mathrm{~V}$, a signal to noise ratio of $\approx 300$ was reached, sufficient to demonstrate the capabilities of TWINSPC here. Figure 3 (a) depicts a photocurrent interferogram in the range from $-0.4 \mathrm{~mm}$ to $0.4 \mathrm{~mm}$. Although the spectrum was recorded over a longer range, the oscillatory signal decays rapidly due to the broad structure of the GaAs absorption spectrum at room temperature. The broad photocurrent spectrum obtained from a Fourier transform of this interferogram is shown in Figure 3 (b). As expected, the signal sets on at the low energy side proportionally to the band edge absorption of GaAs $\left(\alpha_{\text {GaAs }}, E_{g} \approx 1.42 \mathrm{eV}=875 \mathrm{~nm}\right)$ and cuts off on the high energy side proportionally to the intensity of the excitation laser. The insertion of bandpass filters in the excitation beam path artificially limits the spectrum of the illuminating light, which is expected to be visible in the photocurrent spectra. This effect is demonstrated in Figure 3 (b)\&(c), where we have introduced a variety of bandpass filters spanning the recorded photocurrent spectrum. As expected, the narrow band excitation is reflected in the narrow band photocurrent spectra. The interferogram obtained following insertion of an $850 \mathrm{~nm}$ bandpass filter (Thorlabs, FBH850) in the excitation beam path is shown in Figure 3 (c). In the range close to $x=0$, we obtain the same intensity modulation in the photocurrent as in the recorded intensity spectra above (Figure 2 (b)). We compare the Fourier transform of the entire photocurrent interferogram, shown in the inset, with the transmission data obtained from the filter manufacturer in Figure 3 (d) and obtain excellent agreement between the data sets. Specifically, the position and FWHM of both data sets are equal, confirming the calibration procedure and the ability of TWINS-PC to resolve narrow absorption features in this spectral range.
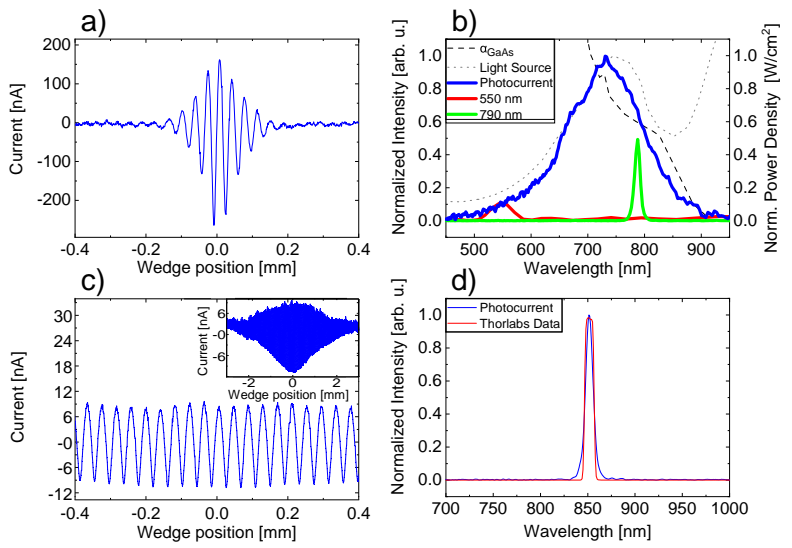

FIG. 3. (a) White light interferogram of the recorded photocurrent as a function of the wedge position in the vicinity of $\tau_{A}=-\tau_{B}$. A constant background is subtracted. (b) Fourier-transformed data showing the different photocurrents in the device with respect to the emission wavelength mirroring the spectrum of the incident light on the sample. (c) Interferogram of a bandpass filter centred at $850 \mathrm{~nm}$ inserted into the excitation beam path resulting in a high-frequency interferogram. The inset shows the interferogram over the entire scanning range. (d) Comparison of the transmission curve provided by the manufacturer of the bandpass filter (Thorlabs BP850) with the FT of the recorded photocurrent.

We can compare the presented interferometric photocurrent spectroscopy setup with a wavelength scanning system requiring a monochromator considering that the minimum number of data points needed to resolve any optical spectra by the two techniques is the same ${ }^{21}$. Namely, if we consider to measure a spectrum with a maximum frequency $v_{\max }$ and a minimum frequency $v_{\min }$ with a desired resolution $\Delta v$, the number of samples is equal to $N=\frac{2\left(v_{\max }-v_{\min }\right)}{\Delta v}$. The main advantages of the interferometric setup are the throughput advantage and the multiplexing advantage. The former is minimal considering a spatially coherent light source, such as in this case, but can be considerable by using a thermal or LED radiation. The latter is related to the ability of the interferometric system to excite the sample with all the spectral components simultaneously. Therefore, if we consider an equal number of data points $\mathrm{N}$, the monochromator employs an excitation intensity that is $\mathrm{N}$ times lower than with the TWINS interferometer. By using the TWINS, this results either in lower integration times to achieve the same signal-to-noise ratio or a better signal-to-noise ratio with the same measurement time. The TWINS interferometer has typically a much higher spectral 
coverage, limited only by the transparency and birefringence of the employed crystals. As an example, the used TWINS interferometer (GEMINI, manufactured by NIREOS) has a working spectral range from $250 \mathrm{~nm}$ to $2300 \mathrm{~nm}$ in a single device. As the maximum excursion of the birefringent wedges is $\pm 12 \mathrm{~mm}$, the maximum time delay between the pulse replicas is $\pm 0.8 \mathrm{ps}$, which equates to a maximum resolution of $\Delta \lambda \approx 2 \mathrm{~nm}$ at a wavelength of $\lambda=700 \mathrm{~nm} .{ }^{19}$ Moreover, since no grating is used, the spectral response is much flatter across all the working spectral range. Another important advantage of the presented photocurrent setup is the ability to increase the spectral resolution by simply increasing the scan range of the measurement without affecting the throughput of the system nor decreasing the aperture of the device. Employing no entrance or exit slits, the shape of the output beam is constant for all measurement applications. This is especially beneficial if one needs to couple the beam into an optical fiber such as in the presented experimental setup. Since the beam is passing through the interferometer without any deviation, the system is easy to align, and the measured spectral properties are less sensitive to misalignment with respect to a grating-based system. Lastly, the optical excitation of the sample and thus the electronic and vibronic state of the specimen is always constant utilizing the TWINS approach, while in grating spectroscopy, local resonances might be excited unbeknownst to the observer.

Figure 4 (a) shows the photocurrent at a fixed wedge position, $x=0$, recorded while the excitation beam is scanned along the grey dashed line shown in Figure 1 (b). We observe a monotonically increasing photocurrent from the positive contact towards the negative contact, as expected. Below, we sketch the related band diagram of our illuminated undoped GaAs-sample $\left(N_{D} \sim 10^{15} \mathrm{~cm}^{-2}\right)$. For a bias voltage of $V_{\text {bias }}=8 \mathrm{~V}$, the depletion region $w$ is approximately $2-3 \mu \mathrm{m}$ wide, indicating that close to the positive contact, flat bands are expected. We highlight the photocurrent spectra at two positions, close to the positive contact and in the middle of the gap between the contacts, as indicated in Figure 1. The total photocurrent signal represents the sum of competing mechanisms such as drift currents under the influence of an electric field that depend on carrier lifetime and mobility, diffusion currents that depend on overall generation rate and resident carrier density as well as photothermal effects. ${ }^{1}$ We expect that close to the positive contact, where the bands are flat, the dominating drift current to be smaller as compared to regions where band bending effects occur in the semiconductor. This is shown in Figure 4 (b), where we plot the PC spectra measured at both positions. The overall current measured in the centre between the contacts is higher compared to the current obtained near the contact, reflecting the stronger dependence of the drift currents on the electric field. The ratio (centre/near contact) of both currents is plotted in the inset. For higher energies, this ratio is constant $\sim 1.6-1.7$. However, in the spectral vicinity of the $\Gamma$-point of the GaAs bandgap, the relative strength of the PCs that were recorded are equal. This measurement exemplifies the capability of our setup for PC spectroscopic mapping combining high spatial and spectral resolution.
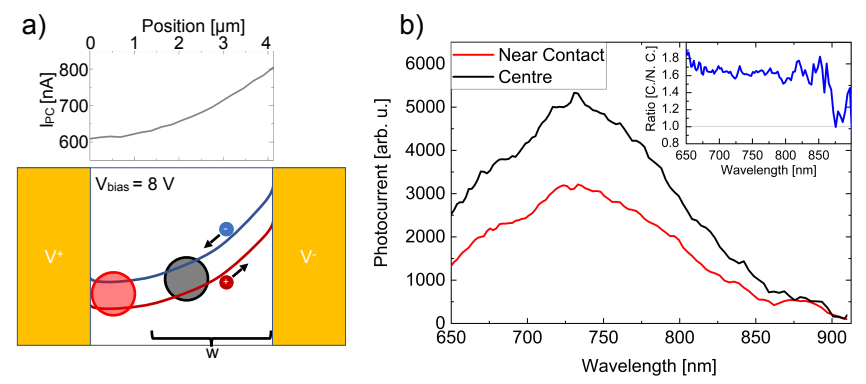

FIG. 4. (a) top: total photocurrent measured at fixed wedge position, $x=0$, when the excitation beam is scanned between the contacts along the grey dashed line shown in Figure 1 (b). bottom: sketch of the band-diagram across the gap between both contacts with applied bias. (b) PC spectra recorded at the marked positions. The overall current detected near the contact is lower in comparison to the current measured in the centre. Inset: Ratio of the current measured in the centre divided by the current obtained near the contact. The ratio is nearly constant for energies well above the GaAs bandgap. Close to the contact, the PC in the spectral vicinity of the $\Gamma$-point of the GaAs bandgap at $875 \mathrm{~nm}$, are equal.

\section{CONCLUSION AND OUTLOOK}

To conclude, we have experimentally validated broadband diffraction-limited photocurrent microscopy following visible excitation. Use of a TWINS birefringent interferometer allows for photocurrent spectroscopy. The TWINS was calibrated by a standard grating-based spectrometer. We further verified the calibration of the TWINS-PC device by performing photocurrent measurements with narrowband excitation selected by a series of interference filters and comparing the retrieved spectra with tabulated ones. Due to the intrinsic phase stability of TWINS, the spectrometer achieves high accuracy without the need for active feedback or path-length tracking, bringing some of the advantages of FT-spectroscopy down to the visible spectral region. For our proof of principle experiments here, we achieved an $\mathrm{S} / \mathrm{N}$ ratio of $\approx 300$. Data averaging and lock-in detection schemes may be employed easily to significantly improve the S/N. We discussed several advantages of the presented technique as compared to a common wavelength scanning system with a monochromator. TWINS can reduce the measurement times and increase the working spectral range considerably. Finally, as reported previously, TWINS can be employed for time-resolved measurements using ultrashort pulses after minor modifications to the interferometer design ${ }^{15}$, highlighting the versatility of this approach $^{22-24}$.

\section{DATA AVAILABILITY STATEMENT}

Data available on request from the authors. 


\section{ACKNOWLEDGEMENT}

We gratefully acknowledge the DFG and European Union for financial support. A. P., D. P. and G. C.'s research was partially funded by the European Union, Horizon 2020 Programme, SimDome Project, Grant Agreement No 814492. J.J.F, J. H and C. H. acknowledge support by Deutsche Forschungsgemeinschaft (DFG, German Research Foundation) through TUM International Graduate School of Science and Engineering (IGSSE), GSC 81 and Germany's Excellence Strategy - EXC 2089/1 (e-conversion). Furthermore, J.J.F. additionally acknowledges the DFG for financial support via EXC 2111/1 (MCQST) and SPP 2244.

We thank Dr. Stefan Krause for his assistance creating Fig. 1.

${ }^{1}$ S. M. Sze and K. Ng. Kwok, Physics of semiconductor devices. John Wiley and Sons, (2006).

${ }^{2}$ J. Nelson, The physics of solar cells, World Scientific Publishing Company, (2003).

${ }^{3} \mathrm{G}$. Bastard, Wave mechanics applied to semiconductor heterostructures, Halsted Press, (1988).

${ }^{4}$ K. F. Mak, C. Lee, J. Hone, J. Shan and T. F. Heinz, Atomically thin $\mathrm{MoS}_{2}$ : a new direct-gap semiconductor. Physical review letters, 105(13), 136805, (2010).

${ }^{5}$ A. R. Klots, et al., Probing excitonic states in suspended two-dimensional semiconductors by photocurrent spectroscopy, Scientific reports $4: 6608$, (2014).

${ }^{6} \mathrm{M}$. Vanecek, and A. Poruba, Fourier-transform photocurrent spectroscopy of microcrystalline silicon for solar cells, Applied Physics Letters 80.5 : 719-721, (2002).

${ }^{7}$ Z. Xiao, Q. Dong, C. Bi, Y. Shao, Y. Yuan and J. Huang, Solvent annealing of perovskite-induced crystal growth for photovoltaic-device efficiency enhancement, Advanced Materials, 26(37), 6503-6509, (2014).

${ }^{8}$ M. Blauth, G. Vest, S. L. Rosemary, M. Prechtl, O. Hartwig, M. Jürgensen, M. Kaniber, A. V. Stier and J. J. Finley, Ultracompact photodetection in atomically thin $\mathrm{MoSe}_{2}$, ACS Photonics, 6(8), 1902-1909, (2019).

${ }^{9}$ S. C. Feifel, K. R. Stieger, H. Lokstein, H. Lux and F. Lisdat, High photocurrent generation by photosystem I on artificial interfaces composed of $\pi$-system-modified graphene, Journal of Materials Chemistry A, 3(23), 12188-12196, (2015).
${ }^{10}$ S. P. Davis, M. C. Abrams, and J. W. Brault, Fourier Transform Spectrometry (Academic Press), (2001).

${ }^{11}$ M. R. Descour, Throughput advantage in imaging Fourier-transform spectrometers, Imaging Spectrometry II. Vol. 2819. International Society for Optics and Photonics, (1996).

${ }^{12}$ P. R. Griffiths, C. T. Foskett, and R. Curbelo. Rapid scan infrared Fourier transform spectroscopy, Applied Spectroscopy Reviews 6.1: 31-77, (1972).

${ }^{13}$ A. Oriana, J. Réhault, F. Preda, D. Polli, and G. Cerullo, Scanning Fourier transform spectrometer in the visible range based on birefringent wedges, J. Opt. Soc. Am. A 33(7), 1415-1420, (2016).

${ }^{14}$ J. Réhault, M. Maiuri, C. Manzoni, D. Brida, J. Helbing, and G. Cerullo, 2D IR spectroscopy with phase-locked pulse pairs from a birefringent delay line, Opt. Express 22(8), 9063-9072, (2014).

${ }^{15}$ J. Réhault, M. Maiuri, A. Oriana, and G. Cerullo, Two-dimensional electronic spectroscopy with birefringent wedges, Rev. Sci. Instrum. 85(12), 123107, (2014).

${ }^{16}$ F. Preda, A. Oriana, J. Réhault, L. Lombardi, A. C. Ferrari, G. Cerullo, and D. Polli, Linear and non-linear spectroscopy by a common-path birefringent interferometer, IEEE J. Sel. Top. Quantum Electron. 23(3), 1-9, (2017).

${ }^{17}$ R. Borrego-Varillas, A. Oriana, L. Ganzer, A. Trifonov, I. Buchvarov, C. Manzoni, and G. Cerullo, Two-dimensional electronic spectroscopy in the ultraviolet by a birefringent delay line, Opt. Express 24(25), 28491-28499, (2016).

${ }^{18}$ E. Thyrhaug, S. Krause, A. Perri, G. Cerullo, D. Polli, T. Vosch and J. Hauer, Single-molecule excitation-emission spectroscopy, Proceedings of the National Academy of Sciences, 116(10), 4064-4069, (2019).

${ }^{19}$ A. Perri, F. Preda, C. D’Andrea, E. Thyrhaug, G. Cerullo, D. Polli, and J. Hauer, Excitation-emission Fourier- transform spectroscopy based on a birefringent interferometer, Opt. Express 25(12), A483-A490, (2017).

${ }^{20}$ T. Zhang, C. Borca, X. Li and S. T. Cundiff, Optical two-dimensional Fourier transform spectroscopy with active interferometric stabilization, Optics Express, 13(19), 7432-7441, (2005).

${ }^{21}$ P. R. Griffiths, J. A. De Haseth, Fourier transform infrared spectrometry, John Wiley and Sons, Mar 16 (2007).

${ }^{22}$ N. Zhou, Z. Ouyang, J. Hu, O. F. Williams, L. Yan, W. You, A. M. Moran, Distinguishing Energy- and Charge-Transfer Processes in Layered Perovskite Quantum Wells with Two-Dimensional Action Spectroscopies, The Journal of Physical Chemistry Letters, 11 (12), 4570-4577, (2020).

${ }^{23}$ P. Gregoire, A. R. S. Kandada, E. Vella, C. Tao, R. Leonelli, C. Silva, Incoherent population mixing contributions to phase-modulation twodimensional coherent excitation spectra, J Chem Phys, 147 (11), (2017).

${ }^{24}$ K. J. Karki, J. R. Widom, J. Seibt, I. Moody, M. C. Lonergan, T. Pullerits, A. H. Marcus, Coherent two-dimensional photocurrent spectroscopy in a $\mathrm{PbS}$ quantum dot photocell, Nat Commun 5, (2014). 